\title{
A Study on Role of Multidetector CT in Characterization of Non Inflammatory Renal Masses
}

\author{
Ritesh Kondeti ${ }^{1}$, Prashanth Thimme Gowda ${ }^{2}$ \\ ${ }^{1}$ Associate Professor, Department of Radiology, Pondicherry Institute of Medical Sciences, Pondicherry, ${ }^{2}$ Associate professor, \\ Department of Radiology, Narayana Medical College, Nellore, Andhra Pradesh, India \\ Corresponding author: Dr. Prashanth Thimme Gowda, Associate Professor, Department of Radiology, Narayana Medical \\ College, Chinthareddypalem, Nellore, Andhra Pradesh, India
}

DOI: 10.21276/ijcmsr.2018.3.3.32

How to cite this article: Ritesh Kondeti, Prashanth Thimme Gowda. A study on role of multidetector CT in characterization of non inflammatory renal masses. International Journal of Contemporary Medicine Surgery and Radiology. 2018;3(3):C146-C149.

\section{A B S T R A C T}

Introduction: Majority of the renal masses are encountered during routine or accidental imaging studies like ultrasonography, computed tomography and Magnetic resonance imaging studies. MDCT remains the single most effective modality in diagnosis of renal non neoplastic and neoplastic masses and staging of renal cell carcinomas. The aim of the present study was to assess the role of multidetector CT in diagnostic evaluation of non inflammatory renal masses.

Material and Methods: A prospective observational two year study approved by ethical committee was conducted. 85 cases with identified renal mass by ultra sonogram were included and MDCT was performed on all the cases in the study.

Results: Out of 85 cases in the study, males were predominant with mean age of $26.21 \pm 2.6$ years. Hematuria was observed in $76.47 \%$ and renal cell carcinoma was commonest. (51.76\%) RCC was most commonly seen in age group of 50-60 years in our study with a mean age of $52.25 \pm 2.5$ years. Most of the cases were in stage III.Transitional renal cell carcinoma was seen in 6 cases. Three cases had metastasis to the liver and 2 cases had associated bladder carcinoma.

Conclusion: MDCT provides preoperative renal vascular status viz, renal artery anatomy, accessory arteries, normal variants, renal vein/IVC invasion and for evaluating the hyperenhancing metastasis in corticomedullary phase. Incidental renal masses with heterogenous enhancement, necrosis, and calcifications are suggestive of renal cell carcinoma.

Key words: Multidetector Computed Tomography, Renal Cell Carcinoma, Transitional Cell Carcinoma.

\section{INTRODUCTION}

Majority of the renal masses are encountered during routine or accidental imaging studies like ultrasonography, computed tomography and Magnetic resonance imaging studies. Most of these are cysts which are asymptomatic or don't require any further management. ${ }^{1}$ However a few of the solid masses are tumours which are benign or malignant. Malignant tumours need to be surgically removed while a few may not require surgical intervention. Differentiation between these masses by imaging studies may avoid unnecessary surgical procedures and economical burden to the cases with renal masses. Accurate delineation of the extent of the renal mass involvement is necessary to ensure best and optimal treatment planning. ${ }^{2}$ A broad improvement in renal imaging modalities ranging from a simple $X$ ray of the abdomen to Multi detector computed tomography has brought an array of changes in the diagnostic perspective of renal masses and management ranging from simple nephrectomy to conservative management options. Due to this rapid improvement in diagnostic modalities in imaging more number of renal masses are being discovered incidentally during routine evaluation and during evaluation of cases with unrelated symptoms. ${ }^{3}$ The wide range of imaging modalities in evaluation of renal masses are plain radiography, Ultrasonography, radionuclide imaging, angiography, CT and MRI. A dedicated renal CT scanning is easily available, economical, quicker than MRI. Multidetector Computed tomography is the latest breakthrough in CT technology which allows rapid renal image acquisition during various phases of contrast enhancement after administration of single bolus dose of contrast material. MDCT remains the single most effective modality in diagnosis of renal non neoplastic and neoplastic masses and staging of renal cell carcinomas. ${ }^{4}$ The aim of the present study was to assess the role of multidetector CT in diagnostic evaluation of non inflammatory renal masses.

\section{MATERIAL AND METHODS}

The present prospective observational study was conducted at a tertiary care hospital of south India by department of radiology in association with department of urology and Nephrology for a period of two years from January 2015 to December 2017. The study was approved by the institutional ethical committee and necessary study protocol was designed and permitted. The study was explained to all the participants in the study and written and informed consent 
was obtained from all the cases. All the cases irrespective of sex and age referred from the departments with suspicious renal mass (based on signs and symptoms) (Palpable renal mass, hematuria, flank pain) and incidental detection of renal mass on USG or CT were included in the study. Patients not willing or consenting for the study, patients allergic to contrast agent, pregnant females, patients with altered renal function tests, were excluded from the study. A detailed clinical history and examination was done by the concerned departmental resident and noted in the protocol questionnaire sheet.

\section{Technique of Ultrasound examination}

The study was carried out by using Philips IU 22 ultrasound machine using probe frequencies ranging from 2.5 to $4 \mathrm{MHZ}$ Philips medical systems. Examination was done with patient lying in supine position, oblique and lateral decubitus positions and findings were noted.

\section{CT protocol}

Multi detector computed tomography was performed using sixty four section MDCT scanner (Philips scanner, Netherlands). A noncontrast and multiphasic post contrast MDCT examination was performed on all subjects of the study. In unenhanced phase scanning was performed from diaphragm down to symphysis pubis and in post contrast phases scanning was performed from diaphragm to iliac crest. The unenhanced phase was performed at $5 \mathrm{~mm}$ thickness and at a speed of $15 \mathrm{~mm}$ rotation. Ultravist $(300 \mathrm{mg} / \mathrm{ml})$ a low osmolar non ionic contrast medium was administered for all cases at $2 \mathrm{ml} / \mathrm{kg}$ body weight at a rate of $4 \mathrm{ml} / \mathrm{s}$ and $150 \mathrm{mmhg}$ using automatic injector. Scanning started immediately using automatic bolus tracking and was triggered 5 seconds after a threshold of $150 \mathrm{HU}$ in the region of interest was reached. All patients underwent scanning during unenhanced phase, cortico medullary phase, Nephrographic phase and Excretory phase.

\section{Assessment of CT features}

The following features of the lesions were assessed: diameter, pattern of enhancement, invasion, presence or absence of calcification, spreading patterns, including perinephric change, venous invasion and lympadenopathy.

\section{STATISTICAL ANALYSIS}

Descriptive statistical analysis was done with the help of Microsoft office 2007. Descriptive statistics like mean and percentages were used for the analysis.

\section{RESULTS}

The present study was carried at a tertiary care hospital of south India which caters the need of around 10 lakh populations with 1050 bedded capacity. A total of 85 cases with suspicious renal masses as per clinical history, accidental observation on imaging (By ultrasonogarm and CT) was included in the study. 65 cases had symptoms and had a clinical examination prior to imaging and 20 cases were incidentally observed on imaging without any symptoms. All the 85 cases were in the age range of 12-76 years and the mean age of the study group was $28.12 \pm 1.8$ years. Males were predominant in the study $(54 / 85,63.53 \%)$ than females $(31 / 85,36.47 \%)$. The mean age

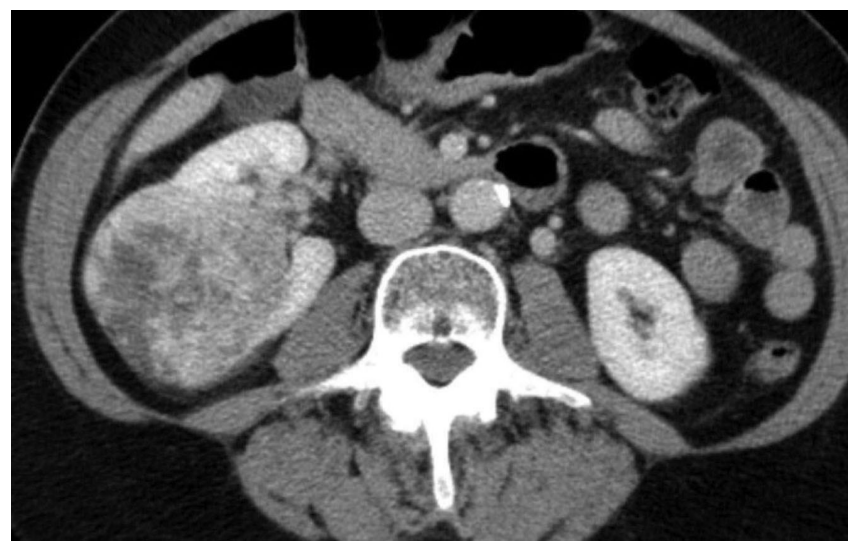

Figure-1: CECT Showing Left Renal Cell carcinoma with Renal vein and IVC extension

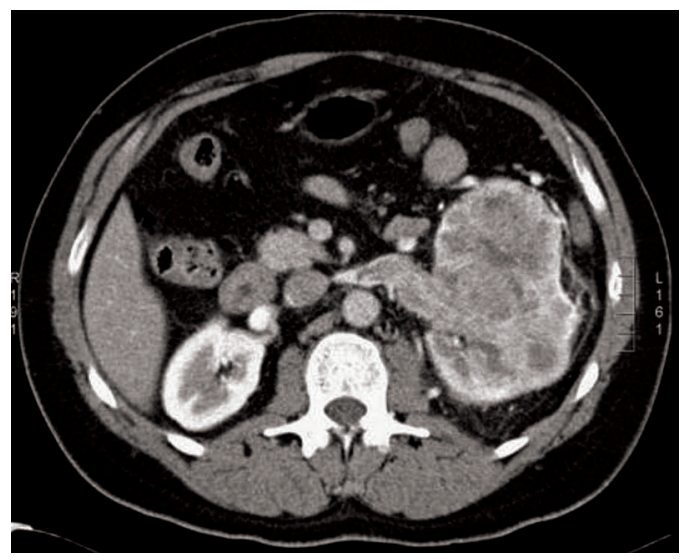

Figure-2: CECT Showing Mid-zone renal cell carcinoma

of males was $26.21 \pm 2.6$ years and females $25.25 \pm 1.4$ years. Majority $(24 / 85,23.24 \%)$ were in the age group of 50-60 years followed in order by $40-50$ years $(22 / 85$. $25.88 \%)$ and the least was $<10$ years $(1 / 85,1.17 \%)$. Hematuria was the most common presenting complaint $(65 / 85,76.47 \%)$ followed by flank pain $(34 / 85,40 \%)$ and palpable mass $(24 / 85,28.23 \%)$. The most common renal mass encountered in our study was renal cell carcinoma (RCC) which was seen in 44 cases $(51.76 \%)$ followed in order by transitional cell carcinoma, Angiomyolipoma in 8 cases (9.41\%). Wilm's tumour was observed in 4 cases $(4.70 \%)$. Less commonly observed were 6 Cystic lesions (Bosniak IV cyst, Bosniak III cyst) renal abscess (6 cases), renal haematoma (5 cases), metastasis (5 cases) and lymphoma (3 cases). RCC was most commonly seen in age group of 50-60 years in our study with a mean age of $52.25 \pm 2.5$ years. Calcification was observed in 12 cases $(12 / 85,14.11 \%)$ with 6 cases with amorphous type and 6 with punctuate in nature. Heterogeneous enhancement was observed on post contrast studies in 69 cases (81.17\%). Cases of renal carcinoma demonstrated altered renal contour and enhancement in cortico medullary and nephrographic phase with washout in excretory phase. [Figure-1] Perirenal fascial extension was observed in 8 cases $(9.41 \%)$, perinephric space extension in 10 cases (11.76\%), ipsilateral adrenal involvement in 12 cases (14.11\%), involvement of renal vein and inferior vena cava was observed in 4 cases (4.70\%). [Figure-2] Pelvicalcyeal involvement in 14 cases (16.47\%) and lymph nodal involvement was seen in 8 cases. (9.41\%) 
Other surrounding structures which were involved were, liver in 5 cases $(5.88 \%)$, abdominal wall involvement in 6 cases (7.05\%) Multiple sites of metastasis was observed in 5 cases (5.88\%) and lung was the most common organ involved. (2.12\%) followed by bone and liver. Regional lymph node metastasis was observed in 14 cases (16.47\%). Heterogenous enhancing metastasis similar to solid organ involvement was the most common pattern observed among 12 cases and hypo dense metastasis in 8 cases of study. Paraaortic and aortocaval nodes of the size more than $10 \mathrm{~mm}$ were taken as positive and there was a direct correlation between size, lymph node involvement and staging of the tumour. Stage III was the most common found in 18 cases followed by Stage IV and II (8 cases).

Transitional renal cell carcinoma was seen in 6 cases $(7.05 \%)$ as heterogeneously enhancing masses which maintained contour along upper ureteral thickening. Three cases had metastasis to the liver and 2 cases had associated bladder carcinoma.

Renal metastasis appeared as multiple heterogeneously enhancing lesions involving bilateral kidneys with bulky necrotic retroperitoneal lymph nodes. Renal metastasis was observed in cases with lung carcinoma.

\section{DISCUSSION}

In the present improved era of diagnostic imaging services, MDCT has definitely improved the ability in differentiating renal masses from usual cystic lesions to benign and malignant tumours and revolutionizing the management in renal masses. MDCT has enabled in clear differentiation and also delineation of renal masses with regard to extension and venous spread, lymph nodal involvement and also local or distant spread of malignant masses. In the present study we assessed the role of MDCT in differentiating and diagnosing and also in preoperative staging of malignancies.

In the present study, 85 cases of suspicious renal masses identified in symptomatic cases or cases incidentally were performed MDCT for further evaluation prospectively. Males were predominant in the study and Renal Cell carcinoma was the most common finding which accounted for $51.76 \%$ of cases which was similar to the finding of Zhang et $a . .^{5}$ In our study, RCC was common in the age group of 50-60 years in our study with a mean age of 52.25 2.5 years which is on par with the report of Wood CG et al. ${ }^{6}$ On USG Heterogenous solid mass appearance was seen in 32 cases and followed by solid mass with cystic appearance in 12 cases. It was distributed in both upper and lower poles in 20 cases and total replacement of kidney by solid mass in 8 cases of RCC. Calcification was observed in 12 cases and varied from punctuate to amorphous pattern and absence of fat attenuation. In 22 cases, mixed areas of heterogenous enhancement, necrosis and cystic changes were observed; 15-20HU difference was taken as significant enhancement between unenhanced and nephrographic phase. These findings of our study were similar to the findings of Agnihotri S et al. ${ }^{8}$

Hematuria was the most common symptom in all the cases presented and was associated with flank pain in majority of cases. Findings of our study were comparable to the findings of Verhoest $\mathrm{G}$ et al who mentioned associated fever and burning micturition in association with palpable solid mass. ${ }^{7}$ In our study, perinephric extension was observed in 10 cases which was defined as presence of perinephric stranding or perinephric collaterals. Other spacial extensions which were observed in our study were Gerota's fascia extension in $8 \%$ of cases, Ipsilateral adrenal involvement in 12 cases and these findings of our study were similar to findings of Chimpri et al. ${ }^{9}$ In our study, involvement of inferior vena cava and renal vein was observed in 4 cases of total cases stating that cases of RCC were staged as T3 and T4. However our study was comparable with the findings of Sheth et al who also reported similar percentage of cases indicating many cases were presented at late stages than early stages of RCC. ${ }^{10}$ In our study most of the cases were diagnosed with stage IV and next with II, similar findings were observed in the study of Das CJ et al. ${ }^{11}$ In our study, we observed a direct correlation between the patients complaint and the staging of the disease and most of the cases which were found incidentally were of stage I only and confining to the kidney only signifying the impact of prognosis in early diagnosed cases incidentally. Regional lymph node metastasis was observed in 14 cases in our study that has not been associated with lymph nodal enlargement N0.In some of the cases nodal enlargement was associated with some benign inflammatory lesions. Jemal Aet al, in 2006 showed, $25-30 \%$ of patients with RCC having metastases at the time of presentation which is comparable with our study. ${ }^{12}$ The degree of enhancement was the most important parameter in differentiation of RCC subtypes. In our study we observed that high attenuation on CT correlates with clear cell carcinomas.

Multiple site metastasis was observed in 5 cases in our study, with involvement of lung, colon and abdominal wall. Lung was the most common site of distant metastasis in our study; similar findings were reported in the study of Zagoria et al with involvement of 8 cases in his study. ${ }^{13}$ In the present study,small hyperenhancing solid organ metastasis in liver was seen only in the corticomedullary phase and became isodense with the liver parenchyma in nephrographic phase implying the need of triple phase CT in evaluation of renal cell carcinoma. Osteolytic metastasis was observed in 2 cases with i case of RCC and other case of transitional cell carcinoma.

In our study, there were 8 cases of transitional renal cell carcinoma, which appeared as mixed echogenic masses involving the kidney on USG. In our study, the main identifying features were focal filling defect in the pelvicalcyeal system, preserved renal shape, absence of cystic or necrotic change and tumour enhancement with extension towards uteropelvic junction. Similar findings were also reported in the study of Catalano $\mathrm{C}$ et al. ${ }^{14}$

Angiomyolipoma was observed in 8 cases which appeared as hyperechoic lesions on USG and with fat attenuation on CT. Presence of fat attenuation was the constant feature differentiating from metastatic lesions.

\section{CONCLUSION}

To conclude, MDCT provides preoperative renal vascular status viz, renal artery anatomy, accessory arteries, normal 
variants, renal vein/IVC invasion and for evaluating the hyperenhancing metastasis in corticomedullary phase. Incidental renal masses with heterogenous enhancement, necrosis, and calcifications are suggestive of renal cell carcinoma. Multiphase CT has a major role in the diagnosis, characterization and staging of solid renal neoplastic masses.

\section{REFERENCES:}

1. Tsili, Athina C, and Maria I Argyropoulou. Advances of Multidetector Computed Tomography in the Characterization and Staging of Renal Cell Carcinoma. World Journal of Radiology 2015;7(6): 110-127.

2. Ching, Boon Chye et al. Differential Radiologic Characteristics of Renal Tumours on Multiphasic Computed Tomography. Singapore Medical Journal 2017;58(5): 262-266.

3. Mancini, Maria Elisabetta et al. Diagnostic Potential of Multidetector Computed Tomography for Characterizing Small Renal Masses. The Scientific World Journal 2015: 476750.

4. G. M. Israel and S. G. Silverman. The incidental renal mass. Radiologic Clinics of North America 2011;49(2):369-383.

5. J. Zhang, R.A. Lefkowitz, N.M. Ishill, L. Wang, C.S Moskowitz, P. Russo, et al. Solid renal cortical tumors: differentiation with CT.Radiology 2007;244(2):494504.

6. Ng CS, Wood CG, Silverman PM, Tannir NM, Tamboli P, Sandler CM. Renal cell carcinoma: diagnosis, staging, and surveillance. AJR Am J Roentgenol. 2008;191(4):1220-32.

7. Verhoest G, Veillard D, Guillé F, De La Taille A, Salomon L, Abbou CC, Valéri A, Lechevallier E, Descotes JL, Lang H, Jacqmin D. Relationship between age at diagnosis and clinicopathologic features of renal cell carcinoma. European Urology. 2007;51(5):1298305.

8. Agnihotri S, Kumar J, Jain M, Kapoor R, Mandhani A. Renal cell carcinoma in India demonstrates early age of onset and a late stage of presentation. Indian J Med Res. 2014;140(5):624-9.

9. Chimpiri AR, Natarajan B. Renal vascular lesions: diagnosis and endovascular management. Semin Intervent Radiol 2009;26(3):253-61.

10. Sheth S, Scatarige J, Horton K, Corl F, Fishman E. Current concepts in the diagnosis and management of renal cell carcinoma: role of multidetector $\mathrm{CT}$ and threedimensional CT. Radiographics 2001;21(4):S237-54.

11. Das CJ,Ahmad Z, Sharma S, Gupta AK. Multimodality imaging of renal inflammatory lesions. World J Radiol 2014;6(11):865-73.

12. Jemal A, Siegel R, Ward E, Murray T, Xu J, Smigal C, et al. Cancer statistics, 2006. CA Cancer J Clin. 2006; 56(2):106-30

13. Zagoria R. Imaging of small renal masses. AJR 2000;175(1):945-55

14. Catalano C, Fraioli F, Laghi A, et al. High resolution multidetector $\mathrm{CT}$ in the preoperative evaluation of patients with renal cell carcinoma. AJR 2003;140(5):8794.

\section{Source of Support: Nil; Conflict of Interest: None}

Submitted: 01-09-2018; Accepted: 10-09-2018; Published online: 18-09-2018 